\title{
Cross-layer Optimization Using Advanced Physical Layer Techniques in Wireless Mesh Networks
}

\author{
Samat Shabdanov, Patrick Mitran, Member, IEEE, Catherine Rosenberg, Fellow, IEEE
}

\begin{abstract}
The objective of this paper is to study the impact of advanced physical layer techniques on the maximum achievable throughput of wireless multihop mesh networks. We formulate a cross-layer optimization framework for the routing and scheduling problem jointly with the following physical layer techniques: successive interference cancellation, superposition coding, dirtypaper coding and their combinations. In the case when each node is enabled with superposition coding, we need to formulate a power allocation subproblem for the optimal power partition of the superimposed signals. We solve these joint problems exactly to compute the maximum achievable throughput in realistic size networks. This allows us to quantify the performance gains obtained by using these techniques (and their combinations). Specifically, we find that the use of dirty-paper coding (only at the gateway) is not justified in networks with mixed uplink and downlink flows. On the other hand, the combination of superposition coding with successive interference outperforms significantly other techniques across all transmission power range for both uplink and downlink flows. We also provide a number of interesting practical insights on throughput improvement by comparing different combinations of these techniques.

Index Terms-Successive interference cancellation, superposition coding, dirty paper coding, multihop networks, cross-layer optimization.
\end{abstract}

\section{INTRODUCTION AND MOTIVATION}

Wireless multihop mesh networks are considered as an attractive solution to offer good throughput, energy, and coverage trade-offs. In order to deliver these trade-offs, there is a need for cross-layer optimization, i.e., the joint optimization of the physical layer parameters (e.g., rate and power), MAC parameters and routing [1]. Two main types of mesh networks have been extensively studied. The first one is based on random access (e.g., Aloha [2] or CSMA/CA) while the second is based on conflict-free scheduling [1], [3], [4]. With the emergence of new standards like LTE and WiMAX, which include conflict-free scheduling as an option, the question of optimally configuring scheduled mesh networks is becoming more and more important.

We consider the case of a managed mesh network composed of mesh routers and one gateway where 1) the mesh routers are fixed and located about 20 meters above ground so that it is reasonable to consider that the channel gains are known and quasi time-invariant and 2) the traffic is heavy enough

Manuscript received October 15, 2011; accepted December 21, 2011. The associate editor coordinating the review of this paper and approving it for publication was Dr. O. Dabeer.

The authors are with the Department of Electrical and Computer Engineering, University of Waterloo, 200 University Ave. West, Waterloo, Ontario, Canada (e-mail: \{sshabdan, pmitran, cath\}@uwaterloo.ca).

This work was supported in part by the Natural Science and Engineering Research Council of Canada (NSERC) and by Research In Motion (RIM)

Digital Object Identifier 10.1109/TWC.2012.111859. that a static (and central) configuration of the scheduling and routing (as well as the physical layer parameters) makes sense. In such a network the traffic flows are from the gateway to the routers (downlink flows) and from the routers to the gateway (uplink flows).

Previous work [4] has shown that significant throughput gains can be obtained in a network configured by jointly optimizing routing and scheduling parameters. In this paper, we present a cross-layer optimization framework for the optimal offline configuration of fixed wireless networks that use advanced physical layer (PHY) techniques. In that sense, this is a pre-planning or planning study. We focus on the following PHY techniques: Successive Interference Cancellation (SIC), Superposition Coding (SPC) and Dirty-paper Coding (DPC) and on some of their combinations. Another recent advanced PHY technique that could be considered is Interference Alignment (IA) [5],[6]. However, we do not include IA in this work due to the fact that it is mainly effective at high transmission powers, and its practical implementation is extremely challenging.

Many advances in physical layer techniques have been proposed in the recent past and they have been studied mostly in an information theory framework. Very few studies, if any, have tried to quantify the gains that these techniques could provide in a realistic network scenario. The principal objective of our offline study is to quantify the gains in maximum achievable throughput that can be obtained by using some of these techniques in wireless mesh networks (WMN) of practical size under a realistic interference model (i.e., the physical interference model [1]). To provide additional practical engineering insights, we also study variants where we restrict the use of these PHY techniques to the gateway.

The throughput limitation of WMNs stems from the halfduplex characteristic of the wireless interfaces and the interference produced by all transmitting nodes which limits the spatial reuse. It was shown in [1],[7],[8] that the gateway is the bottleneck in this kind of network and that without special ways to mitigate these two problems, the per node throughput under a max-min policy is upper bounded by $\frac{r_{m}}{N-1}$ in a network with a single gateway and $N-1$ nodes, where $r_{m}$ is the maximum possible rate for the given set of modulation and coding schemes. Clearly, if each node is able to communicate with the gateway in single hop at rate $r_{m}$ then this upper bound is feasible. It was shown that this bound can typically be reached at much lower transmit power by using multihop communications [4]. In order to increase the pernode throughput beyond this upper bound for the max-min policy, many options are possible. Some of them will impact 
the infrastructure cost, i.e., the channel bandwidth can be increased (or multiple channels can be used) or the number of nodes per gateway can be decreased (i.e., by adding gateways). Other options might affect the complexity of the nodes and/or the gateway but without an increase in capital expenditures. In this paper, we focus on the latter.

By increasing spatial reuse or allowing a node to decode multiple transmissions at the same time, we will be able to increase the maximum achievable per-node throughput. For example, we consider a SIC based PHY technique, first proposed in [9], which is a technique that enables a wireless receiver to decode multiple signals successively to either partially cancel interference [10], [11] or receive more than one packet at a time [12]. We will study the cases where SIC is enabled at all nodes or only at the gateway.

SPC, also initially proposed in [9], is a technique that enables a wireless transmitter to send several signals, possibly intended for different users, as a composite. In order for a user to decode its own signal from such a composite signal, a SIC receiver is necessary. We will study the cases where SPC and SIC are both enabled at all nodes, both only at the gateway, as well as the case when only SPC is enabled at the gateway.

Another advanced technique that we will consider is DPC, first presented in [13]. DPC is a technique used at a transmitter to encode a signal with prior knowledge of the interference at a particular receiver so that, at this receiver, the harmful interference is perfectly mitigated. As a result, it allows a receiver to effectively benefit from an interference free transmission at no extra power cost to the transmitter. We will only study the case when DPC is enabled at the gateway and not in other nodes due to practical implementation challenges that we will discuss later and the high complexity to model DPC at each node. Hence, in the following DPC should be understood as DPC at the gateway only.

The main contributions of the paper can be summarized as follows:

- We formulate joint routing and scheduling flow-based problems to compute the max - min throughput in a WMN when SIC, SPC, DPC or SPC-SIC is enabled at each node. These formulations are based on the physical interference model. On the modeling front, the novelties are 1) in the formulation of the set of ISets for each physical layer technique/combination under study; 2) for superposition coding (SPC), it was necessary to formulate a subproblem to partition the transmission power among the superimposed signals at each transmitter in an optimal fashion.

- These problems are very large scale NP-hard Linear Programs (LP), where the number of variables grows exponentially with the size of a network. We develop efficient tools based on the column generation method to compute exact solutions for realistic size networks.

- Our formulations enable us to obtain not only the exact $\max -\min$ achievable throughput in a given WMN but as well to find an optimal network configuration that achieves this throughput in terms of routing and link scheduling parameters. When studying SPC, we also obtain the optimal power partition for each set of concurrently transmitting links in the optimal schedule.
- We provide practical engineering insights for network operators on how much performance gain can be obtained by using these techniques in isolation or in combinations, and at all nodes or by restricting the use of these techniques only at the gateway. In particular, dirty paper coding has proven very disappointing in terms of throughput improvement. This is due in part to the fact that for DPC, it is necessary to separately optimize uplink and downlink flows. On the other hand, SPC with SIC achieves the theoretical maximum throughput, i.e., $\frac{k \times r_{m}}{N-1}$ for uplink and $\frac{\min (r, k) \times r_{m}}{N-1}$ for downlink flows, where $N-1$ is the number of nodes (not including the gateway) in the network, $r_{m}$ is the highest rate modulation scheme, $k$ is the number of iterations of SIC and $r$ is the number of superimposed signals for SPC (to be described in detail in the sequel) at high power, and provides significant gains at low to medium transmission powers.

The paper is organized as follows. Section II presents the three PHY techniques, namely SIC, SPC and DPC in some details and then discusses related work. Section III describes the system model and the problem formulations when using SIC, SPC, DPC, and SPC-SIC. Section IV presents the numerical results along with the engineering insights.

\section{BACKGROUND AND RELATED WORK}

\section{A. Background}

Interference cancellation schemes can be categorized in three main groups: parallel [14], successive, and a combination of parallel and successive schemes [15]. There are different trade-offs between these schemes in terms of decoding latency, performance and complexity. A parallel scheme is preferable when received powers are somewhat equal and a successive scheme operates at best with unequal received power distribution [12]. Considering the practical complexity of parallel schemes, we consider only the SIC based PHY scheme [16]. A receiver with SIC can decode multiple signals successively to either partially cancel the interference or receive more than one packet at a time. Referring to Fig. 1, without SIC, halfduplex node $b$ cannot receive from both nodes $a$ and $e$ at the same time, and node $d$ can only decode a signal from $c$ if its SINR (Signal to Interference plus Noise Ratio) is greater than a certain threshold. Now with SIC, it might be possible for node $d$ to first decode the strong interfering signal from $a$. Node $d$ can then subtract it from its compound received signal so that it can now decode the signal from $c$. In that case, $d$ has partially canceled the interference to decode the signal from $c$ successfully. With SIC, node $b$ can also decode both signals from $a$ and $e$. Node $b$ first decodes the strong signal from $e$, cancels its interference out to decode successfully the signal from $a$.

SPC is well-known as an efficient technique to increase the throughput of multiuser systems. The idea of SPC is to allow a node to transmit several signals intended for possibly different nodes in a network as a composite signal. When transmitters are enabled with SPC, the use of SIC at the receivers is required for optimal decoding. Considering the practical complexity of both SIC and SPC, SPC is sometimes studied in the literature with a restricted SIC receiver, which is used to 


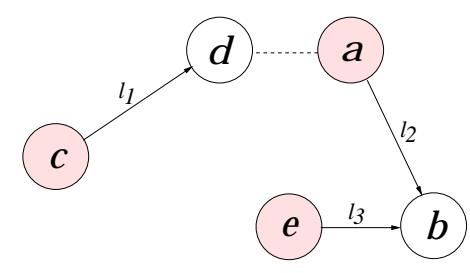

Fig. 1. Illustration of $\operatorname{SIC}(2)$.

decode only superimposed signals from the same source node [17]. We denote the general case of SPC with full capability SIC receivers by $\operatorname{SPC}(r)-\operatorname{SIC}(k)$. In a network where $\operatorname{SPC}(r)$ $\operatorname{SIC}(k)$ is enabled at each node, a transmitter can superimpose up to $r$ signals simultaneously and the receiver can decode a maximum of $k$ signals. Thus the composite transmitted signal is the sum of up to $r$ modulated signals. The restricted variant of superposition coding is referred to as $\operatorname{SPC}(r)$. To explain the scheme in more details, we consider the general case, where a receiver is capable of decoding any signal from any node. Please refer to Fig. 2, where nodes $a$ and $c$ transmit composite signals and node $f$ transmits a direct signal to $g$. All nodes transmit with the same power $P$. The composite signal from node $c$ is the sum of two signals with powers $P\left(\ell_{4}\right)$ and $P\left(\ell_{5}\right)$ destined to nodes $e$ and node $d$ respectively. The composite signal from node $a$ is the sum of two signals with powers $P\left(\ell_{1}\right)$ and $P\left(\ell_{2}\right)$ both destined to node $b$. To allow concurrent transmissions of links $\left\{\ell_{1}, . ., \ell_{5}\right\}$ without harmful interference to each other, the powers at nodes $a$ and $c$ must be partitioned jointly with respect to all link powers. Let us assume that at node $b$ the powers are split such that $P\left(\ell_{1}\right)>P\left(\ell_{2}\right)$ and $P\left(\ell_{1}\right)+P\left(\ell_{2}\right)=P$, then the receiver first decodes a signal over link $\ell_{1}$ and after canceling it out, the receiver at node $b$ decodes a signal over link $\ell_{2}$. At node $c$ the power is split such that $P\left(\ell_{4}\right)>P\left(\ell_{5}\right)$ and $P\left(\ell_{4}\right)+P\left(\ell_{5}\right)=P$, then at nodes $d$ and $g$ the main interferer is the superimposed signal over link $\ell_{4}$ and at node $e$ the main interferer is the direct signal from node $f$. SIC receivers first cancel out their strong interference signals from the compound received signals, and then decode their own signals. This example in Fig. 2 is only possible because we allow SIC receivers to decode the main interference from any node in a network.

Another advanced technique is dirty-paper coding, first presented in [13], and a special case of Gelfand-Pinkser coding for channels with side-information [18]. While details of the implementation of DPC are beyond the scope of this paper, DPC is a pre-coding technique used at a transmitter to encode a signal with prior knowledge of interference at a particular receiver so that at this receiver the harmful interference is "canceled out" without requiring any additional transmission power. As a result, it allows a receiver to effectively benefit from an interference free transmission. However, this is only possible if the transmitter knows the data being sent by other transmitters along with the corresponding channel gains to estimate the interference at a particular receiver. For downlink flows in a scheduled network, this may be reasonably expected for the gateway, as all downlink flows originate from the gateway. For uplink flows, this cannot be expected for any

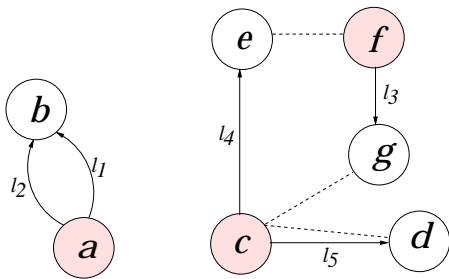

Fig. 2. Illustration of $\operatorname{SPC}(2)-\operatorname{SIC}(2)$.

node in general. For this reason, we will only consider DPC at the gateway (i.e., on the downlink). This, in turn, forces the separation of uplink scheduling from downlink scheduling.

\section{B. Related work}

The original model for the joint routing and scheduling problem for any given wireless network was formulated in [3]. This model provides the optimization framework regardless of the choice of interference model or network utility function for a single channel system. It also provides numerical solutions for lower and upper bounds for the throughput based on a protocol interference model. However, these results are limited to small-sized networks with single power and rate only. The work of [3] was later extended by [1] to include power control and rate adaptation in the joint routing and scheduling problem. [1] also provides a study on an optimal network configuration based on the physical interference model along with exact solutions for the $\max -\min$ achievable throughput in small to medium-size networks. The importance of using the right interference model was studied in [19], where it was concluded that the physical interference model, as opposed to the protocol model, should be used to provide meaningful results. In [4], an efficient enumeration algorithm and a computation model based on the column generation method were proposed to solve the joint routing and scheduling problem for large-sized wireless networks with power control and rate adaption for both max - min and proportional fair throughputs. In [2], the joint routing and medium control problem is formulated for a random access wireless networks.

The work of [20] was among the first to study the impact of SIC on capacity regions in scheduled wireless multihop networks. It presents a mathematical model for finding maximum rate combinations between source and destination nodes in a network. In this paper the interference model is the physical model and the link rates are defined using the continuous Shannon capacity formula. A limitation of this capacity formulation is that numerical results are limited to small-sized (six nodes) networks. In contrast, in our preliminary work [21], the impact of SIC on the throughput of multihop networks was studied for medium-sized networks of up to 25 nodes. We provided a joint routing, scheduling, and multiple decoding SIC problem formulation for $\max$ - min throughput in a wireless multihop network with multiple rates and power capabilities. We have shown that SIC can achieve significant gains at lower powers and overcomes the fundamental throughput limit of $\frac{r_{m}}{N-1}$ discussed above at high powers.

In [17], the joint routing and power allocation with superposition coding is formulated as a non-linear problem 
for a wireless broadcast network (all nodes are transmitting simultaneously) using a continuous link rate model. The power partition of the superimposed signals is jointly optimized with routing for single and multiple power levels at each transmitter. There is no scheduling in this problem since all nodes are transmitting simultaneously, i.e., full-duplex operation is assumed. Another limitation of this work is that the SIC technique is restricted to decode only superimposed signals originating from a common node. Thus, non-SPC and SPC signals originating from other nodes are treated as noise and cannot be canceled out. There is also some interesting work that focuses on the design of practical networks using SPC [22]. Here, the first design of a practical medium access protocol with SPC is presented for wireless mesh networks and average gains in the range of $10 \%$ to $20 \%$ are reported with respect to the standard 802.11 protocol.

Another relevant research area focuses on asymptotic performance bounds in wireless networks (see for example [23]). However, this kind of study does not give any indication on how to optimally configure networks or what performance to expect for a medium size network.

\section{OPTIMIZATION FRAMEWORK}

\section{A. System Model}

We model a wireless multihop network by a graph $\mathcal{G}=$ $(\mathcal{N}, \mathcal{L})$, where $\mathcal{N}$ is a set of nodes and $\mathcal{L}$ is a set of directed links.

Let $\mathcal{F}$ denote a set of flows, where each flow $f \in \mathcal{F}$ is specified by a pair of source and destination nodes $f=\left(f_{o}, f_{d}\right)$, and the rate of a flow $f$ is denoted by $\lambda_{f}$. We do not place any restrictions on node placement and flow patterns at this point, although later we will focus on getting numerical results for mesh networks.

We consider a system in which all nodes use the same transmit power $P$ and the same modulation and coding scheme yielding a unit rate. In order to take parallel links into account when SPC is enabled, we define a link $\ell$ as $\ell=(o(\ell), d(\ell), i)$ where $o(\ell)$ is its origin, $d(\ell)$ is its destination, and $i$ is a unique sequence number to distinguish it from parallel links with the same origin and destination nodes. This sequence number can be omitted for all the cases that do not involve SPC. In the absence of interference, we say that link $\ell \in \mathcal{L}$ is feasible if the signal to noise ratio (SNR) at node $d(\ell)$ meets the threshold requirement $\beta$ for the successful decoding of a modulated signal sent by node $o(\ell)$, i.e.,

$$
\frac{P(\ell) g_{o(\ell), d(\ell)}}{N_{0}} \geq \beta,
$$

where $g_{o(\ell), d(\ell)}$ is the radio channel power gain between nodes $o(\ell)$ and $d(\ell), N_{0}$ is the receiver's background noise, and $P(\ell)=P$ is the transmission power for link $\ell$.

\section{B. Conflict Free Scheduling and ISets}

We define a conflict free schedule as a schedule that only activates at a given time a set of links that produces interference that is not harmful for any receivers, i.e., all the corresponding receivers can successfully decode the signals that are intended for them. A set of links, $s$, for which the interference at all receivers is manageable, is called an ISet, and the set of all ISets is denoted by $\mathcal{I}$ with a suitable subscript as discussed below. Clearly, whether a set $s$ is an ISet will depend on the physical layer techniques that are enabled at the nodes, e.g., we expect that any ISet $s$ when interference is treated as noise would also be an ISet when SIC is enabled but not necessarily vice-versa. Thus, in the remaining subsections, we characterize the conditions for a set $s$ to be an ISet under the various physical layer techniques that we study, and the subscript of $\mathcal{I}$ specifies which physical layer techniques are enabled. We describe SIC for a generic number of iterations $k$ and likewise, SPC for a generic number of superimposed signals $r$ for simplicity of exposition, although in the numerical results section, we take $k \leq 3$ and $r \leq 2$.

A link schedule is specified by a vector $\left[\alpha_{s}\right]_{s \in \mathcal{I}}$, where $\alpha_{s} \geq 0$ represents the fraction of time that ISet $s \in \mathcal{I}$ is scheduled. By activating only ISets the schedule is conflictfree.

\section{ISets when Interference as Noise}

If interference is treated as noise by all nodes in the network then a set of links $s$ can be scheduled concurrently, i.e., is an ISet, if each link $\ell \in s$ obeys the following conditions

[C1] for each $n \in \mathcal{N}, \sum_{\ell \in s} 1_{\{o(\ell)=n\}} \leq 1$,

[C2] for all $\ell_{1}, \ell_{2} \in s$ and $\ell_{1} \neq \ell_{2}$, then $o\left(\ell_{1}\right) \neq d\left(\ell_{2}\right)$,

[C3] for each $n \in \mathcal{N}, \sum_{\ell \in s} 1_{\{d(\ell)=n\}} \leq 1$, as well as the Signal to Interference plus Noise Ratio (SINR) constraint

[S1] For each $\ell \in s$,

$$
\frac{P(\ell) g_{o(\ell), d(\ell)}}{N_{0}+I_{\ell}-P(\ell) g_{o(\ell), d(\ell)}} \geq \beta,
$$

where $I_{\ell}$ is the aggregated received signal power $I_{\ell}=$ $\sum_{\ell^{\prime} \in s} P\left(\ell^{\prime}\right) g_{o\left(\ell^{\prime}\right), d(\ell)}$ at link $\ell$, and $P(\ell)=P$ for all $\ell \in s$.

Requirements $[\mathbf{C} 1]$ and $[\mathbf{C} 3]$ specify that no two distinct links in $s$ can share a source or destination respectively, while $[\mathbf{C 2}]$ is the half-duplex constraint. The constraint $[\mathbf{S 1}]$ specifies that the SINR of each link $\ell \in s$ must be at least the threshold $\beta$. We denote by $\mathcal{I}_{\text {int }}$ the collection of all possible ISets thus described in a network, where interference is treated as noise.

\section{ISets when SIC is Enabled}

We denote by $\operatorname{SIC}(k)$ the case when a node can perform up to $k-1$ rounds of successive interference cancellation, i.e., up to $k$ signals can be decoded at the node. To incorporate $\operatorname{SIC}(k)$ into our system model, we need to formulate under which conditions ISets exist. For each link $\ell \in s$ an ordered set of links $\mathcal{D O}(\ell)=\left(\ell_{1}, \ldots, \ell_{k_{\ell}}\right)$ is defined where $k_{\ell} \leq k$ and $\mathcal{D O}(\ell)$ denotes the decoding order for SIC for link $\ell$ at node $d(\ell)$. Hence, one must have $\ell_{j} \neq \ell_{i}$ for $j \neq i$, as well as $\ell_{j} \in s$, and $\ell=\ell_{k_{\ell}}$. $\operatorname{SIC}(k)$ allows a receiver to decode signals from several nodes at a time or to allow partial decoding of the interference from other nodes [10]. Note that for partial decoding of the interference, the ordered set $\mathcal{D O}(\ell)$ may include links that do not have $d(\ell)$ as a destination. Then, in the network $\mathcal{G}=(\mathcal{N}, \mathcal{L})$ with $r=1$ and $\operatorname{SIC}(k)$, a set of links $s$ is an ISet if it satisfies $[\mathbf{C 1}]$, [C2], as well as 
$\left[\mathbf{C 3}^{\prime}\right]$ for each $n \in \mathcal{N}, \sum_{\ell \in s} 1_{\{d(\ell)=n\}} \leq k$,

$\left[\mathbf{S 1}^{\prime}\right]$ for all $\ell \in s$, there exists a $\mathcal{D O}(\ell)$ such that for each $\ell_{j} \in \mathcal{D O O}(\ell)$

$$
\frac{P\left(\ell_{j}\right) g_{o\left(\ell_{j}\right), d(\ell)}}{N_{0}+I_{\ell}-\sum_{i=1}^{j} P\left(\ell_{i}\right) g_{o\left(\ell_{i}\right), d(\ell)}} \geq \beta
$$

and $P(\ell)=P$ for all $\ell \in s$.

Each link $\ell$ in $s$ must have at least one decoding order set $\mathcal{D O}(\ell)$ to satisfy $\left[\mathbf{S 1}^{\prime}\right]$. For small $k$ the decoding order $\mathcal{D O}(\ell)$ can be found by iteratively checking all possible decoding order sets of length up to $k$. If no decoding order can be found for at least one link in $s$, then the set of links $s$ is not an ISet.

Denote by $\mathcal{I}_{\mathrm{SIC}(k)}$ the collection of all ISets in a network with $\operatorname{SIC}(k)$.

Clearly, since the number of incoming links to any node, including the gateway, is limited to $k$, the $\max -\min$ throughput is now upper bounded by $\frac{k \times r_{m}}{N-1}$ for uplink flows and still by $\frac{r_{m}}{N-1}$ for downlink flows.

We also study the case where SIC is only enabled at the gateway $G$ (in the case of a mesh network). In that case, a set $s$ of links is an ISet if it meets i) [C2] for all links, ii) $[\mathbf{C 1}],[\mathbf{C} 3]$, and $[\mathbf{S 1}]$ for all links $\ell$ such that $d(\ell) \neq G$, and iii) $[\mathbf{C 1}],\left[\mathbf{C 3}^{\prime}\right]$, and $\left[\mathbf{S 1}^{\prime}\right]$ for all links $\ell$ such that $d(\ell)=G$.

\section{E. ISets when SPC and SIC are Enabled}

We denote by $\operatorname{SPC}(r)-\operatorname{SIC}(k)$ a generic SPC scheme, where each transmitter is able to superimpose up to $r$ signals into one composite signal and each receiver is able to decode up to $k$ signals from any node in the network. If a composite signal is the superposition of $r$ signals then it results in $r$ links $\ell_{1}, \ldots, \ell_{r}$ leaving a common source node $n$, and necessarily $\sum_{i=1}^{r} P\left(\ell_{i}\right)=P$, i.e., for these links with a common source node $P\left(\ell_{i}\right) \neq P$. Since, the total transmission power of a composite signal is still $P$, SPC does not introduce additional interference in a network. However, to fully utilize SPC capabilities, the transmission powers over superimposed links must be allocated optimally and jointly between all SPC destination nodes to maximize the spatial reuse in a network. At first glance, it is not trivial to find such a jointly optimal power allocation since we also allow $\operatorname{SIC}(k)$ at each node.

If in a network $\mathcal{G}=(\mathcal{N}, \mathcal{L})$, each node is enabled with $\operatorname{SPC}(r)-\operatorname{SIC}(k)$, then a set of links $s$ is an ISet if it satisfies $[\mathbf{C 2}],\left[\mathbf{C 3}^{\prime}\right],\left[\mathbf{S 1}^{\prime}\right]$ and

$\left[\mathbf{C 1}^{\prime}\right]$ for each $n \in \mathcal{N}, \sum_{\ell \in s} 1_{\{o(\ell)=n\}} \leq r$,

[C4] for each $n \in \mathcal{N}, \sum_{\ell \in s: o(\ell)=n} P(\ell)=P$.

For a given set of links $s$, let $\mathbf{P}_{\mathbf{s}}=[P(\ell)]_{\ell \in s}$ be a vector of power associations for all the links in $s$. Each link $\ell$ in $s$ is either a direct link with $P(\ell)=P$ or a superimposed link with $P(\ell)<P$. It is easy to check if a set of links $s$ is an ISet for all conditions except $\left[\mathbf{S 1}^{\prime}\right]$. To check the feasibility of links for $\left[\mathbf{S 1}^{\prime}\right]$, it is necessary to enumerate all inequalities $\left[\mathbf{S 1}^{\prime}\right]$ for each possible decoding order $\mathcal{D O}(\ell)$ of length up to $k$ for each link in $s$. The set $s$ is an ISet if we can find at least one decoding order $\mathcal{D O}(\ell)$ for each link that satisfies $\left[\mathbf{S 1}^{\prime}\right]$ for a common power vector $\mathbf{P}_{\mathbf{S}}$.

We address this SINR feasibility $\left[\mathbf{S 1}^{\prime}\right]$ of an ISet by formulating a power allocation subproblem. The purpose of this subproblem is to find a vector power $\mathbf{P}_{\mathbf{s}}$ and a decoding order $\mathcal{D O}(\ell)$ that satisfy the condition $\left[\mathbf{S 1}^{\prime}\right]$ for each link $\ell$ in $s$.

For a fixed set of links $s$, denote by $\mathcal{D}(\ell)$ the collection of all possible decoding order sets of length up to $k$ for link $\ell$. Let us define a binary variable $a_{m, \ell}$ as follows:

$a_{m, \ell}= \begin{cases}1, & \text { if } m \text { is a feasible decoding order for link } \ell \\ 0, & \text { else, }\end{cases}$

where $m \in \mathcal{D}(\ell)$ and $\ell \in \mathcal{L}$.

If $s$ satisfies the node constraints given in $[\mathbf{C 2}],\left[\mathbf{C 3}^{\prime}\right]$, $\left[\mathbf{C 1}^{\prime}\right]$, then the optimal power allocation subproblem for $\mathbf{P}_{\mathbf{s}}$ can be formulated as follows:

$$
\begin{array}{cl}
\Delta=\min _{\mathbf{P}_{\mathbf{s}}, \boldsymbol{a}, \boldsymbol{\phi}} \sum_{\substack{m \in \mathcal{D}(\ell) \\
\ell_{j} \in m \\
\ell \in s}} \phi_{m, \ell_{j}, \ell} & \\
P\left(\ell_{j}\right) g_{o\left(\ell_{j}\right), d(\ell)}-\beta\left(N_{0}+I_{\ell}\right) a_{m, \ell}+ & \\
\beta \sum_{i=1}^{j} P\left(\ell_{i}\right) g_{o\left(\ell_{i}\right), d(\ell)}+\phi_{m, \ell_{j}, \ell} \geq 0 & \forall \ell \in s, \forall \ell_{j} \in m \\
\sum_{\substack{\ell^{\prime} \in s \\
o\left(\ell^{\prime}\right)=o(\ell)}} P\left(\ell^{\prime}\right)=P & \forall \ell \in \mathcal{D}(\ell) \\
\sum_{m \in \mathcal{D}(\ell)} a_{m, \ell} \geq 1 & \forall \ell \in s \\
a_{m, \ell} \in\{0,1\} & \forall \ell \in s \\
\mathbf{P}_{\mathbf{s}}, \boldsymbol{\phi} \geq \mathbf{0} & \forall m \in \mathcal{D}(\ell)
\end{array}
$$

In the formulation above, we denote by $\phi$ the vector of all artificial variables $\phi_{m, \ell_{j}, \ell}$ and by $\boldsymbol{a}$ the vector of all binary variables $a_{m, \ell}$. Constraints (5) are the SINR conditions for each link in $s$. Constraints (6) specifies the SPC power constraints, and (7) specifies that each link $\ell$ should have at least one decoding order $\mathcal{D O}(\ell)$ that is feasible. The $\phi_{m, \ell_{j}, \ell}$ are indicator variables in the sense that if all $\phi_{m, \ell_{j}, \ell}=0$, then there is a feasible solution to $\left[\mathbf{S 1}^{\prime}\right]$. Specifically, if the solution to the subproblem results in $\Delta=0$ ( $\Delta$ is the value of the objective function), then there must be a common power vector $\mathbf{P}_{\mathbf{s}}$, and for each link $\ell$ in $s$, there is a decoding order $\mathcal{D O}(\ell)$ such that $a_{\mathcal{D O}(\ell), \ell}=1$. Hence, for these decoding orders and since all $\phi_{m, \ell_{j}, \ell}=0,(5)$ implies condition $\left[\mathbf{S 1}^{\prime}\right]$ is satisfied. Conversely, if $\Delta>0$, then there does not exist a power vector $\mathbf{P}_{\mathbf{s}}$, and decoding orders $\mathcal{D O}(\ell)$ that satisfy $\left[\mathbf{S 1}^{\prime}\right]$ for $s$. In practice, due to numerical scaling issues, to check if an optimal power allocation is feasible for $\left[\mathbf{S 1}^{\prime}\right]$, we look for $\Delta \in[-\delta, \delta]$, where $\delta$ is a small value (of the order of $10^{-12}$ ).

Denote by $\mathcal{I}_{\mathrm{SPC}(r)-\mathrm{SIC}(k)}$ the collection of all ISets in a network with $\operatorname{SPC}(r)-\operatorname{SIC}(k)$. For $\operatorname{SPC}(r)-\operatorname{SIC}(k)$, since the maximum number of incoming links and outgoing links from any node is $k$ and $r$ respectively, the $\max$ - min throughput for uplink flows is upper bounded by $\frac{k \times r_{m}}{N-1}$, whereas for downlink flows it is upper bounded by $\frac{\min (r, k) \times r_{m}}{N-1}$.

For comparison purposes with [17], we also consider the case of $\operatorname{SPC}(r)$ where SIC is only employed to cancel interference from a single composite source at a time, i.e., $\left[\mathbf{C 1}^{\prime}\right]$, 
$[\mathbf{C 2}],\left[\mathbf{C 3}^{\prime}\right],[\mathbf{C 4}]$ and $\left[\mathbf{S 1}^{\prime}\right]$ hold, where $\mathcal{D O}(\ell)$ in $\left[\mathbf{S 1}^{\prime}\right]$ is limited to links from the same source as link $\ell$. Denote by $\mathcal{I}_{\mathrm{SPC}(\mathrm{r})}$ the collection of all ISets in a network using $\operatorname{SPC}(r)$ with restricted variant of $\operatorname{SIC}(k)$.

\section{F. ISets when DPC is Enabled}

In the context of a scheduled wireless mesh network, a gateway on the downlink has perfect knowledge of the data being sent in a network since all packets originate from gateway itself. With appropriate feedback, it can be made aware of any links along with their channel gains. Thus, in a scheduled network, the gateway is able to estimate the interfering signal at any node from other active links for downlink flows only. For uplink flows, in general, it is not possible for any node to be aware of the interfering signals as uplink flows do not have a common node of origin. Thus, for DPC, we separate the scheduling of uplink and downlink flows. For downlink flow scheduling, links that originate from the gateway are interference free using DPC, while all other links will be subject to interference. For uplink flows, all links are subject to interference.

Thus, if $\mathcal{I}_{\mathrm{DPC}}$ is the collection of all ISets with DPC, then each ISet $s \in \mathcal{I}_{\mathrm{DPC}}$ must satisfy the requirements of $[\mathbf{C 1}]$, $[\mathbf{C 2}],[\mathbf{C 3}]$ and in addition the SINR condition $[\mathbf{S 1}]$ if $o(\ell) \neq$ $G$ or eq. (1) for $o(\ell)=G$. $\mathcal{I}_{\mathrm{DPC}}$ is only used to schedule downlink flows. Uplink flows are scheduled using $\mathcal{I}_{\text {int }}$, and the two schedules are combined by time sharing so as to maximize the minimum flow rate over all uplink and downlink flows.

\section{G. Problem Formulation}

In this section, we provide a cross-layer optimization framework for the joint routing and scheduling (JRS) problem with the PHY techniques (and their combinations) introduced above. The JRS problem is formulated for the $\max -\min$ throughput since it was shown in [4] that the max - min throughput is a reasonable objective for a managed wireless mesh network (WMN).

Given a set of nodes $\mathcal{N}$ and a set of directed links $\mathcal{L}$ and a set of flows $\mathcal{F}$ denote by $x_{f}(\ell)$ the amount of flow $f$ transmitted over a link $\ell$ and by $\boldsymbol{x}_{f}=\left[x_{f}(\ell)\right]_{\ell \in \mathcal{L}}$ the routing vector of a flow $f$. Define the variable $\lambda_{f}$ to be the rate of flow $f$. Also, to simplify notation use vectors $\boldsymbol{x}=\left[\boldsymbol{x}_{f}\right]_{f \in \mathcal{F}}$, $\boldsymbol{\lambda}=\left[\lambda_{f}\right]_{f \in \mathcal{F}}$ and $\boldsymbol{\alpha}=\left[\alpha_{s}\right]_{s \in \mathcal{I}}$, where $\alpha_{s}$ was defined as the proportion of time ISet $s$ is scheduled.

Denote by $\mathcal{I}$ a set of ISets that can be constructed based on the conditions described above for each physical layer technique, i.e., $\mathcal{I}$ is either $\mathcal{I}_{\text {int }}, \mathcal{I}_{\mathrm{SIC}(k)}, \mathcal{I}_{\mathrm{SPC}(r)-\mathrm{SIC}(k)}, \mathcal{I}_{\mathrm{SPC}(r)}$.

Then, given $\mathcal{I}$, the JRS problem for the max - min throughput optimization over all flows can be formulated in a generic form as follows [1]:

$$
\begin{gathered}
\max _{\boldsymbol{\alpha}, \boldsymbol{x}, \boldsymbol{\lambda}} \lambda \\
\sum_{\substack{\ell \in \mathcal{L} \\
o(\ell)=n}} x_{f}(\ell)-\sum_{\substack{\ell \in \mathcal{L} \\
d(\ell)=n}} x_{f}(\ell)=\left\{\begin{array}{lll}
\lambda_{f}, & n=f_{o} & \forall n \in \mathcal{N} \\
-\lambda_{f}, & n=f_{d} & \forall f \in \mathcal{F} \\
0, & \text { else }
\end{array}\right. \\
\sum_{f \in \mathcal{F}} x_{f}(\ell) \leq \sum_{s \in \mathcal{I}} \alpha_{s} \mathbf{1}_{\{\ell \in s\}}
\end{gathered}
$$

$$
\begin{aligned}
& \sum_{s \in \mathcal{I}} \alpha_{s}=1 \\
& \lambda_{f} \geq \lambda \\
& \boldsymbol{\alpha}, \boldsymbol{x}, \boldsymbol{\lambda}, \lambda \geq 0
\end{aligned}
$$

Condition (11) specifies the flow conservation constraints. Link scheduling constraints are given in (12) and (13). Specifically, (12) defines the link capacity constraint, i.e., the aggregated amount of all flows over the link cannot exceed its scheduled capacity (recall that we have one modulation/coding scheme of unit rate). Constraint (13) states that ISets must be scheduled over a unit period of time. Note that $\left[\alpha_{s}\right]_{s \in \mathcal{I}}$ is a function of $\mathcal{I}$ and so are (12) and (13).

By solving this problem, we are not only able to compute the $\max -\min$ throughput that can be obtained using these physical layer techniques but also an optimal network configuration in terms of routing and ISets scheduling. In the case of the SPC technique, we also obtain the optimal power allocation for each ISet.

For DPC, we solve the JRS problem twice: first for downlink flows only using $\mathcal{I}_{\mathrm{DPC}}$, and then for uplink flows only using $\mathcal{I}_{\text {int }}$. These two schedules are then combined using time sharing so as to maximize the minimum flow rate over all uplink and downlink flows.

Although, the problem in (10)-(15) is a LP, it is a very large scale NP-hard problem, where the number of variables grows exponentially with the size of the network. The maximum number of links is in the order of $O\left(N^{2}\right)$ and the number of ISets is in the order of $O\left(|\mathcal{L}|^{M}\right)$, where $M$ is the maximum ISet size. It is not tractable to directly enumerate all ISets as this requires testing all $2^{|\mathcal{L}|}-1$ elements of the power set of $\mathcal{L}$ to check if they are ISets. In addition, in the case of SPC, for each element in the power set, a subproblem must be solved to check if there exists an optimal power allocation for SPC signals. This subproblem is a binary problem for which it is necessity to construct all possible decoding orders for each link. Thus, an enumeration search is not a viable technique .

To compute exact solutions for realistic size networks, we developed computational tools based on the column generation method (or revised simplex method). The problem is solved iteratively on a subset of ISets for a current solution and dual variables. At each new iteration a selected subset of ISets is added to the problem based on the reduced costs

$$
-\left(\zeta+\sum_{\ell \in s} v_{\ell}\right)
$$

of each ISet $s$, where $v_{\ell}$ and $\zeta$ are the dual variables for (12) and (13), respectively. If, at a new iteration, no feasible ISet with a strictly positive reduced cost can be found, then the current solution is optimal. Using this approach we are able to avoid the enumeration of all ISets and to solve the problems within reasonable time.

\section{NUMERICAL RESUlTS}

In this section, we provide exact numerical solutions for medium size wireless mesh networks (WMN) with a total of $N=20$ nodes, where $N-1$ nodes are placed uniformly at 


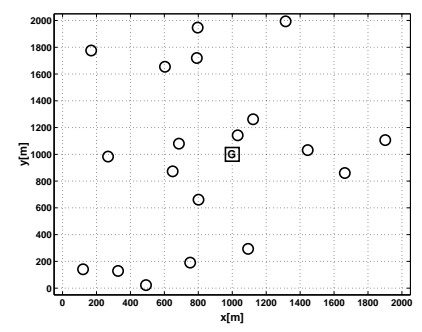

(a) Net-A

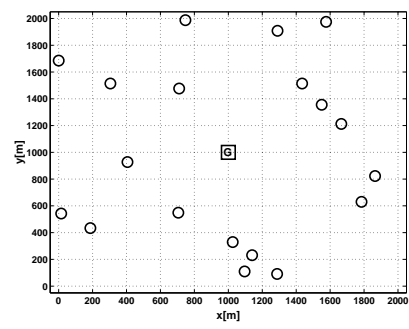

(b) Net-B
Fig. 3. Placement of nodes

random in a $2 \mathrm{~km}$ by $2 \mathrm{~km}$ square, and a gateway node $G$ is placed in the center of the square. The flow pattern for mesh networks is typically from each node to the gateway (uplink) and from the gateway to each node (downlink). We will show our results in terms of node max - min throughput denoted as $\Lambda$ where $\Lambda=\lambda$ (where $\lambda$ is the solution to the JRS problem with the corresponding PHY technique) in a network with only uplink (resp. downlink) flows, and $\Lambda=2 \lambda$ when there are an uplink flow and a downlink flow per node.

We assume that each node uses a fixed transmit power budget $P$ and the same single modulation scheme yielding a normalized rate with a corresponding SINR threshold of $\beta=6.4 \mathrm{~dB}$.

Without loss of generality, we model the channel gains by the path-loss

$$
g_{o(\ell), d(\ell)}=\left(\frac{d_{o(\ell), d(\ell)}}{d_{0}}\right)^{-\eta}
$$

where $d_{0}$ is the near-field crossover distance, $\eta$ is the path loss exponent and $d_{o(\ell), d(\ell)}$ is the distance between a transmitting node $o(\ell)$ and a receiving node $d(\ell)$. Note that our framework is general enough to accommodate any quasi-static channel model. For all our results for $\max -\min$ per node throughputs, we use the following physical layer parameters: $N_{0}=-100 \mathrm{dBm}, d_{0}=10 \mathrm{~m}$ and $\eta=3$. In the following, $P_{S H}$ is the minimum power required for all nodes to communicate with the gateway in a single hop. We have studied multiple realizations and at first for comparative analysis, we show the throughput improvements using advanced physical layer techniques in two selected networks Net-A and Net-B, which are shown in Fig. 3(a) and in Fig. 3(b), respectively. Net-A and Net-B were selected because among the many realizations that we have performed, Net-A in general had the largest performance gains, while Net-B had the lowest.

In Fig. 4, we show $\Lambda$ as a function of the transmission power $P$ when the flow pattern is uplink-only for networks Net-A and Net-B. We label $J R S_{\text {int }}$ the case where ISets $\mathcal{I}_{\text {int }}$ are used, i.e., all interference is treated as noise. In that case, the maximum achievable throughput is bounded to $1 /(N-$ $1)=0.0526$, which is achieved in both networks at much lower powers than $P_{S H}$ due to the multihop communication [4]. We label $\operatorname{SIC}(2)$ and $\operatorname{SIC}(3)$, respectively the cases where SIC with $k=2$ and $k=3$ are enabled at each node. The theoretical maximum throughput for $\operatorname{SIC}(2)$ is $2 /(N-1)=$ 0.1053 and for $\operatorname{SIC}(3)$ it is $3 /(N-1)=0.1579$, and yet at no transmission power is this performance for $\operatorname{SIC}(3)$ achieved as

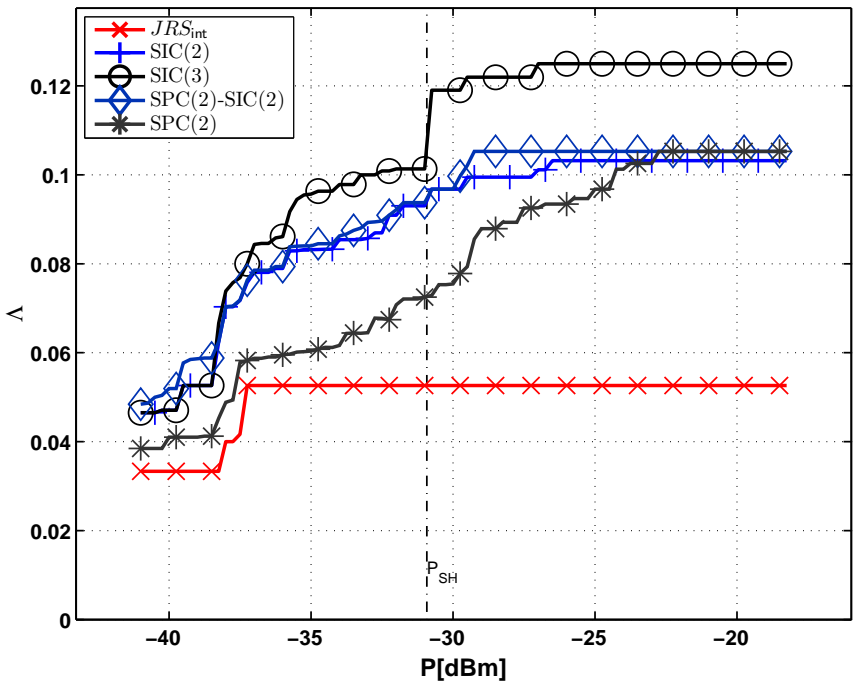

(a) Net-A

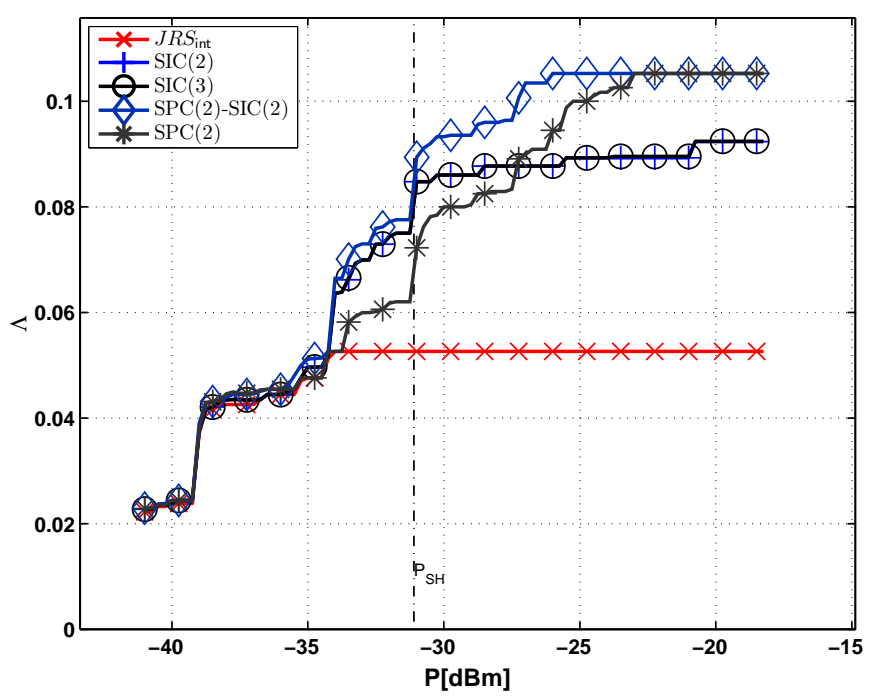

(b) Net-B

Fig. 4. $\max -\min$ throughput vs transmission power $\mathrm{P}$, uplink flows

the node placements of Net-A and Net-B do not permit three simultaneous transmissions to the gateway at all times.

We also notice that at low transmission powers, $\operatorname{SIC}(3)$ does not provide significant improvement compared to $\operatorname{SIC}(2)$. For the network Net-B, SIC does not provide any gains at all in the low power regime due to the fact that with this network topology with path-loss, the channel gains do not provide a sufficient unequal received power distribution. On the other hand, for the network Net-A, SIC provides large gains across all transmission power range by allowing the gateway (and other nodes) to receive multiple transmissions simultaneously, and thus, increasing the size of ISets and the cardinality of $\mathcal{I}_{\mathrm{SIC}(k)}$.

In the case of $\operatorname{SPC}(2)-\operatorname{SIC}(2)$ (labeled as such in the figures), we observe that, in both networks, the maximum throughput of $2 /(N-1)$ can be obtained at powers $P \geq P_{S H}$. In fact, this is to be expected, as at sufficiently high power, each node can then transmit in single hop fashion to the gateway two parallel links with superposition coding, thus 
doubling the rate. If we now focus on low transmission powers in both networks, we observe that $\operatorname{SPC}(2)-\operatorname{SIC}(2)$ does not outperform $\operatorname{SIC}(2)$ significantly as in this regime the achievable throughput is bounded by the decoding capabilities of the gateway node. We also show results for $\operatorname{SPC}(2)$ (i.e., with restricted $\operatorname{SIC}(2)$ decoders). This type of SPC model is used in [17]. Our aim by considering $\operatorname{SPC}(2)$ is to show that such an approach has significant performance penalty. As shown in Fig. 4, in both networks, SPC(2) underperforms significantly at low and medium transmission powers. Note that we do not show results for DPC in Fig. 4 since we consider only uplink flows.

In Fig. 5, we show $\Lambda$ in networks Net-A and Net-B as a function of the transmission power $P$ when the flow pattern is downlink-only. In this case, the optimal $\max -\min$ rate is limited by the transmission capabilities of the gateway $G$, which, if in continuous operation, can produce a $\max -\min$ throughput of $1 /(N-1)$ per node except when SPC is enabled. Although, the use of SIC can potentially improve throughput at low transmission powers, it cannot overcome the bound of $1 /(N-1)$ on the downlink regardless of the number of decodings $k$ as the gateway is the bottleneck. However, with the use of $\operatorname{SPC}(2)-\operatorname{SIC}(2)$, it achieves the theoretical maximum throughput of $2 /(N-1)$ for both networks. This combination of SPC(2)-SIC(2) allows two (or more) outgoing transmissions from any node simultaneously, and at high powers allows for parallel links between two nodes. Thus, SPC with full capability SIC may double the throughput in both uplink and downlink. Comparing with $\operatorname{SPC}(2)$, these results show that this variant of SPC operates near the performance of $\operatorname{SPC}(2)-\operatorname{SIC}(2)$ for downlink flows. We also show results labeled as DPC (i.e., DPC is enabled at the gateway). As shown in Fig. 5, DPC does not provide any gain for Net-A, and only marginal gains for $N e t-B$.

In Fig. 6, we consider the case where there are uplink and downlink flows. We jointly optimize uplink and downlink flows for networks Net-A and Net-B for all cases except DPC for which uplink and downlink flows are optimized separately. With the use of $\operatorname{SPC}(2)-\operatorname{SIC}(2)$, the theoretical maximum throughput of $2 /(N-1)$ is achieved for both Net-A and Net-B.

To investigate further, we consider the cases where we restrict the use of $\operatorname{SIC}(2), \operatorname{SIC}(3)$ and $\operatorname{SPC}$ at the gateway only, and denote these results by $\operatorname{SIC}(2) @ G, \operatorname{SIC}(3) @ G$ and $\operatorname{SPC}(2) @ G$-SIC $(2)$, respectively. In the last variant $\operatorname{SPC}(2) @ G$-SIC $(2), \operatorname{SPC}(2)$ is enabled only at the gateway and $\operatorname{SIC}(2)$ is enabled at each node. We aim to show that by enabling these techniques at the gateway only, we can achieve significant throughput improvements in a network with uplink and downlink flow pattern. As seen in Fig. 6, in both networks, these restricted variants show significant gains at medium to high powers when compared to the case when no advanced PHY techniques are enabled in any node. This indicates that at medium to high power the $\max -\min$ throughput is mainly limited by the transmission capabilities of the gateway. Interestingly, the $\operatorname{SPC}(2) @ G+\operatorname{SIC}(2)$ variant shows almost no decrease in terms of throughput compared with $\operatorname{SPC}(2)-\operatorname{SIC}(2)$. It is because on the uplink all the gains are attributed to $\operatorname{SIC}(2)$ and on the downlink all gains are attributed to $\operatorname{SPC}(2)$ by allowing two transmissions from the

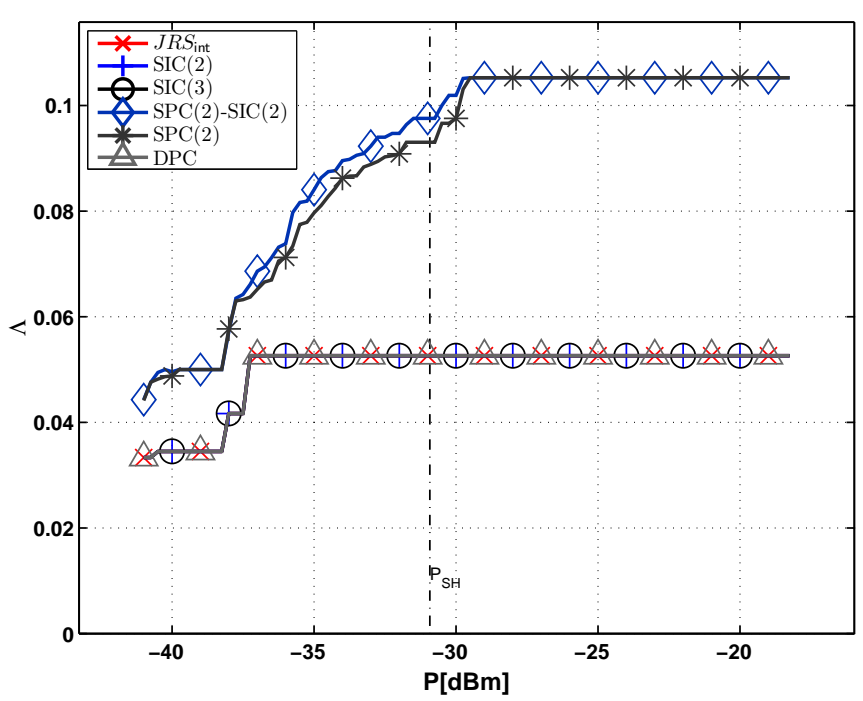

(a) Net-A

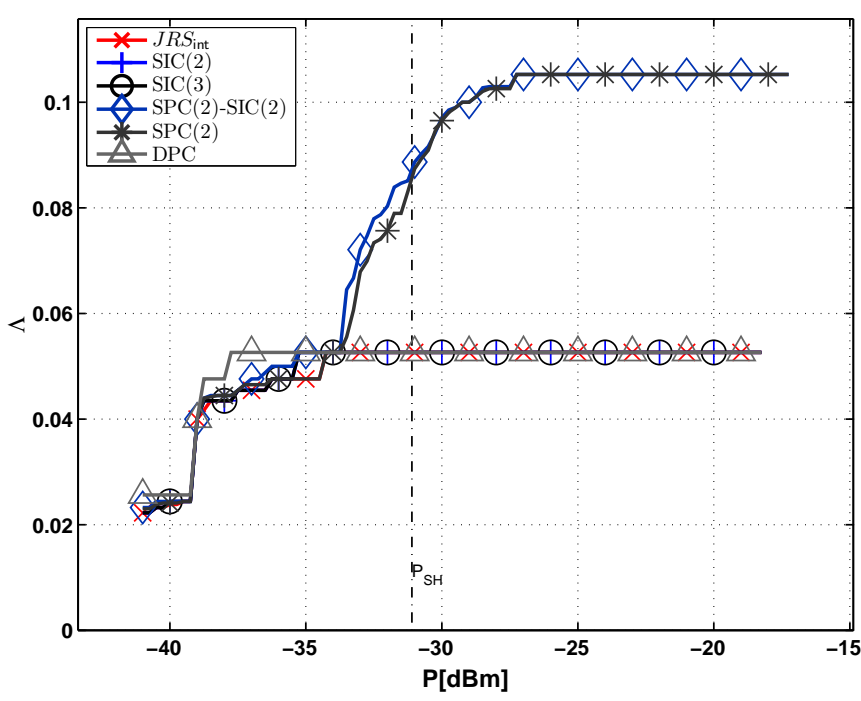

(b) Net-B

Fig. 5. $\max -\min$ throughput vs transmission power $\mathrm{P}$, downlink flows.

gateway.

In the case of DPC, the per-node throughput is obtained by time-sharing separately optimized uplink and downlink schedules such that overall uplink and downlink flow rates are equal. Thus, the overall flow rate is $\Lambda=2 \frac{\lambda_{u l} \lambda_{d l}}{\lambda_{u l}+\lambda_{d l}}$, where $\lambda_{u l}$ and $\lambda_{d l}$ are the uplink and downlink flow rates obtained by separate optimization. Fig. 6 shows that DPC is not justified in a network with mixed uplink and downlink flows and separate optimization of flows can even result in throughput losses as seen in Net-A.

Although not presented here, results for the combination DPC-SPC(2)-SIC(2) have also been obtained. These results showed that for a case with uplink and downlink flows, DPC in combination with $\operatorname{SPC}(2)-\operatorname{SIC}(2)$ does not improve throughput compared to $\operatorname{SPC}(2)-\operatorname{SIC}(2)$ alone. The reason again is the necessity to separately optimize uplink flows and downlink flows when employing DPC. While for downlink flows, DPC in combination with $\operatorname{SPC}(2)-\operatorname{SIC}(2)$ did provide some gain over $\operatorname{SPC}(2)-\operatorname{SIC}(2)$ alone, the cost of time-sharing 


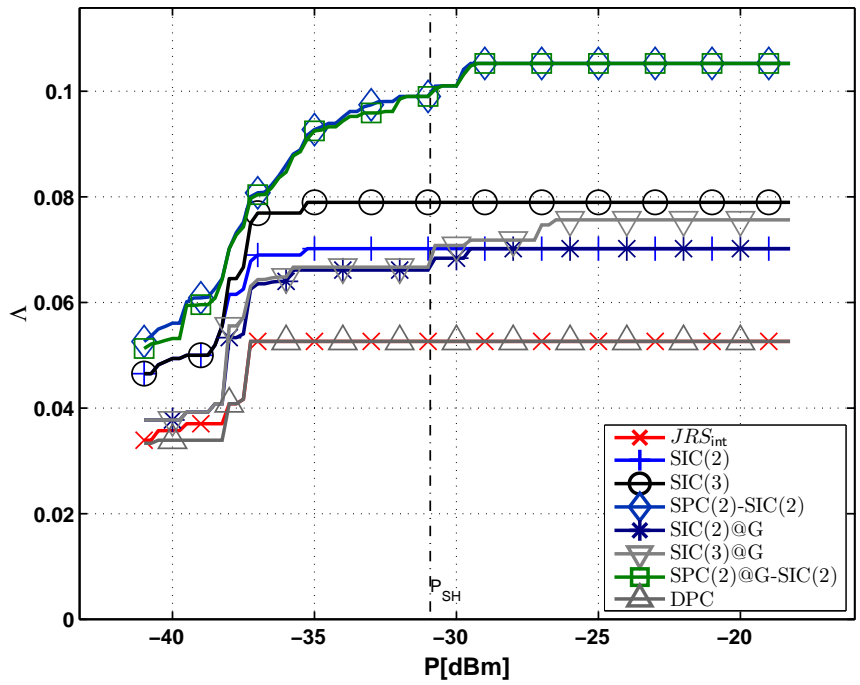

(a) Net-A

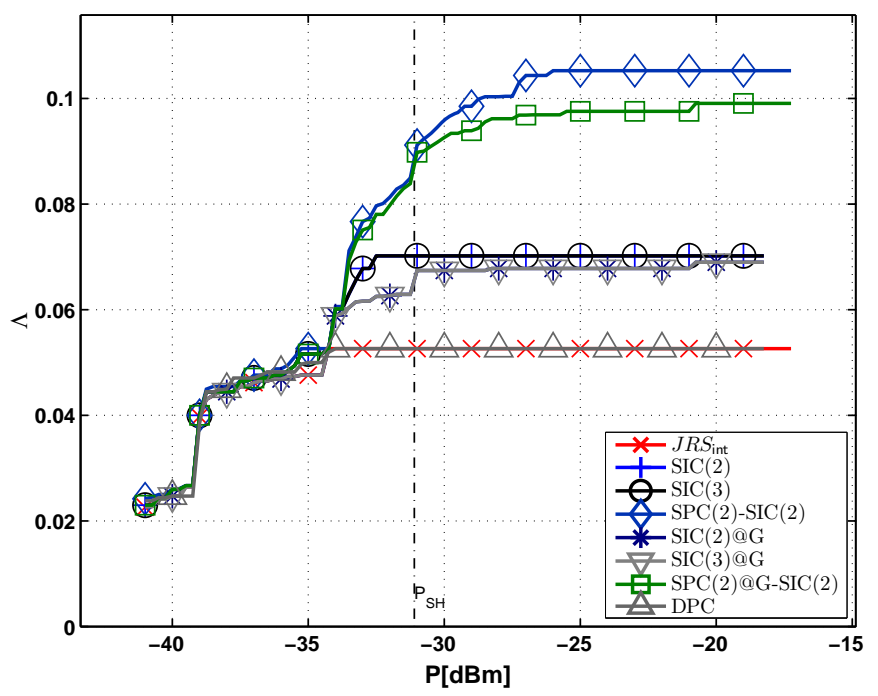

(b) Net-B

Fig. 6. $\max -\min$ throughput vs transmission power P, uplink+downlink flows

separately optimized uplink and downlink flows was greater. Therefore, we conclude that the use of DPC is not worth the implementation complexity when DPC is enabled only at the gateway in networks with mixed uplink and downlink flows.

In Fig. 7, we show the relative gains of each technique with respect to the baseline results for the $J R S_{\text {int }}$ case as a function of the transmission power $P$ in networks with uplink and downlink flows. The relative gains of each technique enabled at each node are computed as gain $(P)=\frac{\bar{\Lambda}(P)-\bar{\Lambda}(P)_{J}}{\bar{\Lambda}(P)_{J}} 100 \%$, where $\bar{\Lambda}(P)$ is the averaged throughput at power $P$ over 10 random network realizations and $\bar{\Lambda}_{J}(P)$ is the averaged throughput at power $P$ for the $J R S_{\text {int }}$ case. Each network realization has the same number of nodes with the gateway located in the center. We provide these results to show general "average" trends for throughput in random topology networks. The results for DPC show that it provides no gains and even underperforms compared to the $J R S_{\text {int }}$ case where uplink

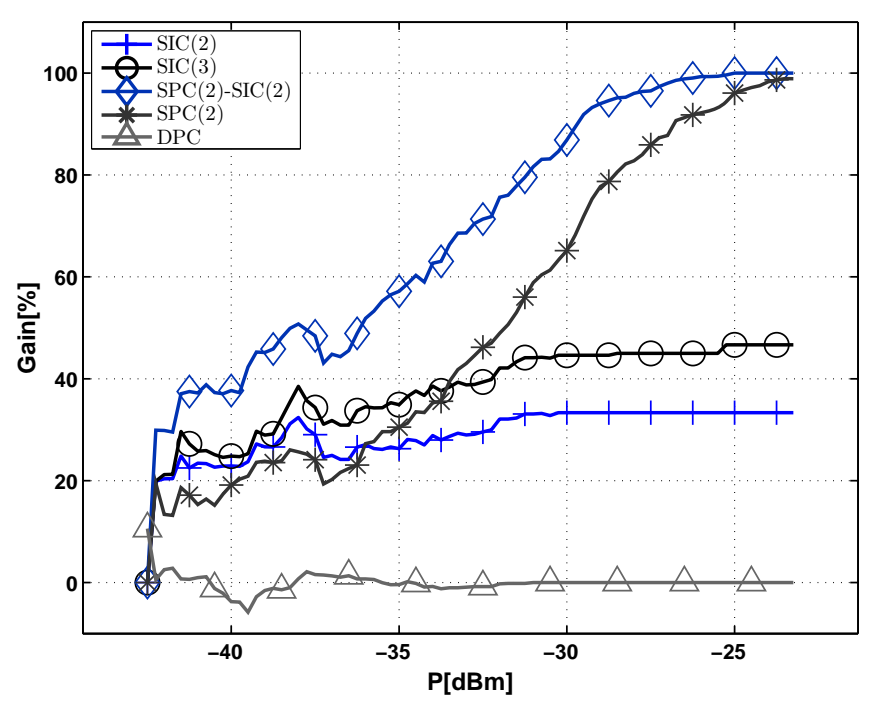

Fig. 7. Relative gain vs transmission power $P$, uplink+downlink flows

and downlink flows are jointly optimized. For $\operatorname{SIC}(2)$ and SIC(3), high throughput gains are obtained across the entire range power. However, even $\operatorname{SIC}(3)$ lags the throughput gains that can be offered by $\operatorname{SPC}(2)-\operatorname{SIC}(2)$. As shown in Fig. 7, $\operatorname{SPC}(2)-\operatorname{SIC}(2)$ provides the maximum possible throughput increase from $100 \%$ at high powers, $60-80 \%$ at medium powers and even outperforms SIC-3 at low power regime with gains of up to $40 \%$.

We also computed results for $\beta=1 \mathrm{~dB}$ (recall that $\beta$ is the modulation SINR threshold). We observe the same general trends except that networks can be connected at about $5 \mathrm{~dB}$ lower power and higher throughput gains can be obtained with SIC and SPC-SIC for Net-A at all transmission powers and for Net-B at high powers.

\section{CONCLUSiOnS}

In this paper, we have provided an optimization framework to determine the achievable throughput in a wireless mesh network that employs successive interference cancellation, superposition coding and dirty-paper coding. With the use of our framework, we have shown the following: 1) enabling SIC allows a network to improve significantly the per node maxmin throughput across the entire transmission power range; 2) SPC with full SIC capabilities significantly outperforms any other technique for both uplink and downlink flows and achieves the maximum theoretical throughput at high power; 3) DPC enabled only at a gateway is not justified for use in the network with uplink and downlink flow patterns due its limited use only on the downlink; 4) implementing SPC-SIC at the gateway brings significant performance gains.

\section{REFERENCES}

[1] A. Karnik, A. Iyer, and C. Rosenberg, "Throughput-optimal configuration of fixed wireless networks," IEEE/ACM Trans. Netw., vol. 16, no. 5, pp. 1161-1174, 2008.

[2] M. Uddin, C. Rosenberg, W. Zhuang, and A. Girard, "Joint configuration of routing and medium access parameters in wireless networks," in Proc. IEEE Globecom 2009, Dec. 2009, pp. 1-8. 
[3] K. Jain, J. Padhye, V. N. Padmanabhan, and L. Qiu, "Impact of interference on multi-hop wireless network performance," in Proc. MobiCom2003: Mobile Comput. Netw., 2003, pp. 66-80.

[4] J. Luo, C. Rosenberg, and A. Girard, "Engineering wireless mesh networks: Joint scheduling, routing, power control, and rate adaptation," IEEE/ACM Trans. Netw., vol. 18, pp. 1387-1400, Oct. 2010.

[5] V. R. Cadambe, S. A. Jafar, and S. Shamai, "Interference alignment on the deterministic channel and application to fully connected Gaussian interference networks," IEEE Trans. Inf. Theory, vol. 55, pp. 269-274, Jan. 2009.

[6] F. Baccelli, A. E. Gamal, and D. Tse, "Interference networks with pointto-point codes," IEEE Trans. Inf. Theory, vol. 57, no. 5, pp. 2582-2596, May 2011.

[7] J. Jun and M. Sichitiu, "The nominal capacity of wireless mesh networks," IEEE Wireless Commun., vol. 10, no. 5, pp. 8- 14, Oct. 2003.

[8] B. Aoun and R. Boutaba, "Maxmin fair capacity of wireless mesh networks," in Proc. IEEE Conf. Mobile Ad-hoc Sensor Syst., Oct. 2006.

[9] T. Cover, "Broadcast channels," IEEE Trans. Inf. Theory, vol. IT-18, no. 1 , pp. 2-14, 1972.

[10] T. S. Han and K. Kobayashi, "A new achievable rate region for the interference channel," IEEE Trans. Inf. Theory, vol. 27, no. 1, pp. 4960, 1981.

[11] R. Etkin, D. Tse, and H. Wang, "Gaussian interference channel capacity to within one bit," IEEE Trans. Inf. Theory, vol. 54, no. 12, pp. 55345562, Dec. 2008.

[12] J. G. Andrews, "Interference cancellation for cellular systems: A contemporary overview," IEEE Wireless Commun. Mag., vol. 12, pp. 19-29, Apr. 2005.

[13] M. Costa, "Writing on dirty paper," IEEE Trans. Inf. Theory, vol. 29, no. 3, pp. 439-441, May 1983.

[14] M. Varanasi and B. Aazhang, "Multistage detection in asynchronous code-division multiple-access communications," IEEE Trans. Commun., vol. 38, no. 4, pp. 509-519, Apr. 1990.

[15] M. K. Varanasi, "Group detection for synchronous gaussian codedivision multiple access channels," IEEE Trans. Inf. Theory, vol. 41, no. 4, pp. 1083-1096, 1995.

[16] P. Patel and J. Holtzman, "Analysis of a simple successive interference cancellation scheme in a DS/CDMA," IEEE J. Sel. Areas Commun., vol. 12, pp. 796-807, 1994.

[17] R. H. Gohary and T. J. Willink, "Joint routing and resource allocation via superposition coding for wireless data networks," IEEE Trans. Signal Process., vol. 58, pp. 6387-6399, Dec. 2010.

[18] S. I. Gel'fand and M. S. Pinsker, "Coding for channels with random parameters," Probl. Contr. Inf. Theory, vol. 9, no. 1, pp. 19-31, 1980.

[19] A. Iyer, C. Rosenberg, and A. Karnik, "What is the right model for wireless channel interference?" IEEE Trans. Wireless Commun., vol. 8, no. 5 , pp. 2662-2671, 2009.

[20] S. Toumpis and A. Goldsmith, "Capacity regions for wireless ad hoc networks," IEEE Trans. Wireless Commun., vol. 2, no. 4, pp. 736-748, July 2003.

[21] P. Mitran, C. Rosenberg, and S. Shabdanov, "Throughput optimization in wireless multihop networks with successive interference cancellation," in Proc. Wireless Telecommun. Symp.(WTS), Apr. 2011, pp. 1-7.

[22] L. E. Li, R. Alimi, R. Ramjee, J. Shi, Y. Sun, H. Viswanathan, and Y. R. Yang, "Superposition coding for wireless mesh networks," in Proc. Conf. MobiCom '07. ACM, 2007, pp. 330-333.
[23] P. Gupta and P. Kumar, "The capacity of wireless networks," IEEE Trans. Inf. Theory, vol. 34, no. 5, 2000.

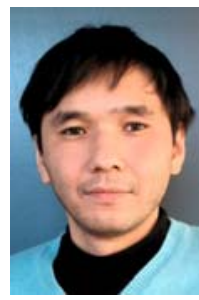

Samat Shabdanov received the B.Sc. with distinction in computer engineering from the University ITMO, St. Petersburg, Russia in 1999 and M.Sc. in communications technology from the University of Ulm, Germany in 2002. He worked as a research assistant at the Institute of Communications and Navigation, German Aerospace Center, Oberpfaffenhofen from 2002 to 2003 and at the Communications Technology Institute, Technical University of Dortmund from 2005 to 2006. Since 2007, he has been pursuing towards the $\mathrm{Ph} . \mathrm{D}$. degree in electrical engineering at the University of Waterloo, Canada. His research activities focus on wireless networking: resource allocation, scheduling, routing, crosslayer design and optimization.

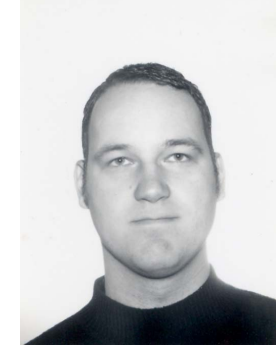

Patrick Mitran (S'01, M'07) received the Bachelor's and Master's degrees in electrical engineering, in 2001 and 2002, respectively, from McGill University, Montreal, PQ, Canada, and the Ph.D. degree from the Division of Engineering and Applied Sciences, Harvard University, Cambridge, MA in 2006. In 2005, he interned as a research scientist for Intel Corporation in the Radio Communications Lab. In 2006-07 he was an applied mathematics lecturer in the School of Engineering and Applied Sciences, Harvard University. Since fall 2007, he is with the Department of Electrical and Computer Engineering at the University of Waterloo at the rank of Assistant Professor.

Currently he is interested in the study of cooperation and cognition in wireless networks from signal processing, coding theory and information theory perspectives.

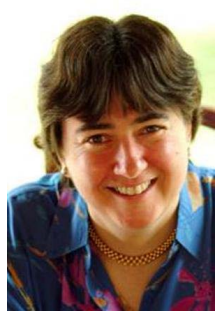

Catherine Rosenberg was educated in France (Ecole Nationale Supérieure des Télécommunications de Bretagne, Diplôme d'Ingénieur in EE in 1983 and University of Paris, Orsay, Doctorat en Sciences in CS in 1986) and in the USA (UCLA, MS in CS in 1984), Dr. Rosenberg has worked in several countries including USA, UK, Canada, France and India. In particular, she worked for Nortel Networks in the UK, AT\&T Bell Laboratories in the USA, Alcatel in France and taught at Purdue University (USA) and Ecole Polytechnique of Montreal (Canada). Since 2004, Dr. Rosenberg is a faculty member at the University of Waterloo where she now holds a Tier 1 Canada Research Chair in the Future Internet. Her research interests are broadly in networking with currently an emphasis in wireless networking and in traffic engineering (Quality of Service, Network Design, and Routing). She has authored over 100 papers and has been awarded eight patents in the USA. She is a fellow of the IEEE. More information can be found at http://ece.uwaterloo.ca/ cath/. 